\title{
The quality of internet information relating to oral leukoplakia
}

\author{
Pía López-Jornet, Fabio Camacho-Alonso
}

Department of Oral Medicine, Faculty of de Medicine and Dentistry, University of Murcia, Spain

Correspondence:

Dentistry Clinic Universitary

Hospital Morales Meseguer (Second Floor)

Medicina Bucal

Avda/Marqués de los Vélez $s / n$

30008 - Murcia (Spain)

fcamacho@um.es

Received: $15 / 10 / 2009$

Accepted: 24/12/2009
López-Jornet P, Camacho-Alonso F. The quality of internet information relating to oral leukoplakia. Med Oral Patol Oral Cir Bucal. 2010 Sep 1;15 (5):e727-31.

http://www.medicinaoral.com/medoralfree01/v15i5/medoralv15i5p727.pdf

\begin{tabular}{|l|}
\hline Article Number: $3236 \quad$ http://www.medicinaoral.com/ \\
C Medicina Oral S. L. C.I.F. B 96689336-pISSN 1698-4447 - eISSN: 1698-6946 \\
eMail: medicina@medicinaoral.com \\
Indexed in: \\
-SCI EXPANDED \\
-JOURNAL CITATION REPORTS \\
-Index Medicus / MEDLINE / PubMed \\
-EMBASE, Excerpta Medica \\
-SCOPUS \\
-Indice Médico Español \\
\hline
\end{tabular}

\begin{abstract}
Objectives: The Internet provides great opportunities for patient healthcare education, but poses risks that inaccurate, outdated or harmful information will be disseminated. Our objective was determine the quality of the information available on the internet in relation to oral leukoplakia.

Study design: Sites were identified using 3 search engines (Google, Yahoo and MSN), and the search term "oral leukoplakia". The first 100 consecutive sites in each search were visited and classified. The websites were evaluated for quality of content by using the validated DISCERN rating instrument and the JAMA benchmarks; the existence of the Health on the Net $(\mathrm{HON})$ seal was also registered.

Results: The Google search yielded 54.300 sites for oral leukoplakia, while Yahoo yielded 243.000 and MSN 103.000. We reviewed 21 Google websites, 20 Yahoo websites and 19 MSN. Based on the JAMA benchmarks, only 4 sites (19.1\%) met the four criteria in the Google search, versus 2 sites (10\%) in the Yahoo and 5 (26.3\%) in MSN. With the DISCERN instrument, no site obtained the maximum score, with Google 13 sites presented serious deficiencies (61.9\%), in the Yahoo search 14 (70\%), and in MSN 15 (78.9\%). Lastly, 4 of the Google sites (19.1\%), four of the Yahoo sites (20\%) and 2 (10.5\%) MSN sites presented the HON seal.

Conclusions: The quality of the health care information related to oral leukoplakia on the internet is poor.
\end{abstract}

Key words: Oral leukoplakia, Internet, treatment, quality of information.

\section{Introduction}

The leucoplakia is the most frequently occurring precancerous lesion. Although the rates of malignant transformation vary between studies, probably as a result of the different criteria used in diagnosis and the follow up intervals, the morbidity and mortality associated with oral cancer make leucoplakia an important health problem (1-4). The World Health Organization (WHO) first defined oral leukoplakia as a white patch or plaque that could not be characterized clinically or pathologically as any other disease. At a 1983 international seminar, the following definition was proposed (1): Leukoplakia is a whitish patch or plaque that cannot be characterized clinically or pathologically as any other disease and is not associated with any physical or chemical causative agent, except the use of tobacco. A more recent WHO workshop recommended abandoning the distinction between the terms "potentially malignant lesions" and 
"potentially malignant conditions" and to use the term "potentially malignant disorders" instead.

The treatment of leucoplasia is based on the premise of early detection and the active management of patients to prevent the development of the squamous cell carcinoma (3). The protocols for managing this clinical entitu are directed at reducing the risk of malignant transformation. The most frequently used being observation and follow up of the lesions, modification of smoking and drinking habits of the patient, chemoprotection (retinoids and carotenes), extirpation (cold scalpel, laser, cryosurgery), photodynamic therapy and the topical application of bleomycin and 5-fluorouracil $(4,5)$.

The accurate information concerning the risks and benefits of medications is important for the patient safety. However, communication between health professionals and patients is inherently problematic.

From the perspective of a health professional, a number of barriers exist, including the use of technical terminology, the volume of information to be conveyed, time constraints and lack of familiarity with the information on the patient's part (6-9).

The Internet has a number of advantages over other formats for communicating health information. It allows rapid and easy access to a vast store of medical knowledge previously only available in specialist libraries. Access is available all day, every day. It is also able to provide links to related websites, expanding the speed and range of information acquired in a single search. The greatest barrier to the Internet reaching its potential to inform health care is not the difficulty in finding information but, rather, finding valid, reliable information. Many factors affect the quality of web based information. Proprietors of health-care websites are competing for sales and market share, which can often lead to selective disclosure of evidence and the presentation of inaccurate information (10-14).

Our objective was to evaluate the quality of web pages on oral leukoplakia available on the Internet, using three general search engines: Google, Yahoo and MSN. The websites were evaluated for quality of content by using the validated DISCERN rating instrument and the JAMA benchmarks; finally, the existence of the Health on the Net (HON) seal was also registered.

\section{Material and Methods}

Sites on the Internet were identified using 3 search engines: Google (www.google.com), Yahoo (www.yahoo. com) and MSN (www.msn.com); all accessed on 9 April, 2009, and the search term "oral leukoplakia". The first 100 consecutive sites in each search were visited and classified. The search was not restricted in terms of file format or domain. The search was limited to the English language. Duplicate sites were excluded, as were non-operative sites or sites with denied direct ac- cess through password requirement, book review sites, or sites offering journal abstracts and those sites that did not offer information on oral leukoplakia. The quality of website information was assessed by 2 independent reviewers and when any difference in opinion occurred, the final score was decided by consensus after discussion. The relevant websites were saved on DVD for later analysis to avoid any changes that may be made to the selected websites during the period of analysis.

The sites were classified in terms of affiliation (commercial, non-profit organization, university or medical center and government), and specialization (exclusively related or partially related to oral leukoplakia). The type of content was classified as corresponding to medical facts, clinical trials, questions and answers and human experiences of interest.

The quality of information of the selected websites was assessed using criteria known as the JAMA benchmarks (12). These are a display of the authorship of medical content, display of attribution or references, disclosure of ownership and display of currency (date of update).

We also registered the existence of the Health on the Net (HON) Foundation seal, this is a code of conduct for medical and health care sites (15). The HON contemplates compliance with eight basic quality criteria about the information for user: 1. authorship, 2. complementarity; 3. privacy, 4. attribution of references and currency, 5. justifiability, 6. author transparency, 7. Sponsor transparency (financial disclosure) and 8. honesty in advertising policy.

The DISCERN instrument, in turn, is a validated rating tool of 16 questions that is freely available online (www. discern.org.uk) and can be used by health consumers or professionals alike (16). The 16 questions are organized into three sections as follows: questions 1-8 address the reliability of the publication and help users to decide whether it can be trusted as a source of information relating to treatment choice, questions 9-15 address specific details of the information relating to treatment alternatives. The question 16 corresponds to the global quality assessment at the end of the instrument. Each question (including question number 16) is scored on a scale of $1-5$ (where $1=$ the publication is poor and $5=$ the publication is of good quality).

\section{Statistical analysis}

Data were analysed using SPSS 12.0 statistics program (SPSS ${ }^{\circ}$ Inc, Chicago, IL, USA). A descriptive study was made of each variable. The Kolmogorov-Smirnov normality test and Levene variance homogeneity test were applied, and the data showed a skewed distribution, and analysed using a non-parametric ranking test. The associations between the different qualitative variables were studied using Pearson's $\chi^{2}$ test. We used the Kruskal-Wallis test (for more than two samples) and the 
Mann-Whitney U-test for two independent samples, for quantitative variables. A probability of less than $\mathrm{p}$-valor $\leq 0.05$.was accepted as significant.

\section{Results}

The Google search yielded 54.300 sites for oral leukoplakia, Yahoo 243.000 and MSN 103.000. The first 100 consecutive sites in each search were examined. The Google search yielded: 4 duplicate sites, 1 site was nonoperative, 64 corresponded to book reviews or journal abstracts, 3 were not relevant to oral leukoplakia and 7 were in languages other than English; finally, we reviewed 21 websites in total. In turn, the Yahoo search yielded: 7 duplicate sites, 1 non-operative site, 59 corresponded to book reviews or journal abstracts, 8 were not relevant and 5 were in languages other than English. We reviewed 20 websites in total. Finally, the MSN search yielded: 4 were duplicate sites, 1 sites was nonoperative, 72 corresponded to book reviews or journal abstracts and 4 were not relevant to oral leukoplakia. We reviewed 19 websites in total. Seven web pages coincided in all three search engines.
The web pages were classified according to the different treatments proposed for oral leukoplakia: life style healthful, nutritional supplements, drugs and surgical treatments (Table 1). These websites were also classified according to affiliation, specialization and type of content (Table 2).

The (Table 3) shows the results according to the JAMA benchmarks or criteria, no statistically significant differences were found between the three search engines. As regards the web pages satisfying the 4 JAMA criteria, only $4(19.1 \%)$ in Google, 2 in Yahoo (10\%) and 5 in MSN (26.3\%).

In relation to the Health on the Net (HON) seal, 4 Google sites (19.1\%), 4 Yahoo sites (20\%) and 2 in MSN (10.5\%) presented this certification. No statistically significant differences were observed between the 3 search engines $(\mathrm{p}=0.683)$.

In the case of the DISCERN instrument, none of the reviewed websites yielded a maximum score with any of the search engines (Table 4). With Google, 13 sites presented serious deficiencies $(61.9 \%)$, while 8 had moderate limitations (38\%). In the case of the Yahoo search, 14 sites presented serious deficiencies (70\%) and MSN $15(78.9 \%)$.

Table 1. Classification of treatments for oral leucokoplakia proposed in the three search engines.

\begin{tabular}{|l|c|c|c|c|}
\hline \multicolumn{1}{|c|}{ Treatments } & $\begin{array}{c}\text { Total } \mathbf{( n = 6 0 )} \\
\mathbf{n}(\mathbf{\%})\end{array}$ & $\begin{array}{c}\text { Google } \\
(\mathbf{n = 2 1 )} \mathbf{n}(\mathbf{\%})\end{array}$ & $\begin{array}{c}\text { Yahoo (n=20) } \\
\mathbf{n}(\mathbf{\%})\end{array}$ & $\begin{array}{c}\text { MSN Search } \\
(\mathbf{n = 1 9 )} \mathbf{n}(\mathbf{\%})\end{array}$ \\
\hline LSH $^{*}+\mathrm{NS}^{* *}$ & $3(5.0)$ & $2(9.5)$ & $1(5)$ & $0(0)$ \\
\hline LSH + Drugs & $17(28.3)$ & $7(33.3)$ & $7(35)$ & $3(15.7)$ \\
\hline LSH + Surgical & $10(16.7)$ & $3(14.3)$ & $3(15)$ & $4(21.1)$ \\
\hline LSH + Drugs + Surgical & $21(35.0)$ & $8(38.1)$ & $5(25)$ & $8(42.1)$ \\
\hline LSH + NS + Drugs + Surgical & $3(5.0)$ & $1(4,8)$ & $2(10)$ & $0(0)$ \\
\hline LSH + NS + Drugs & $5(8.3)$ & $0(0)$ & $2(10)$ & $3(15.7)$ \\
\hline LSH only & $1(1.7)$ & $0(0)$ & $0(0)$ & $1(5.4)$ \\
\hline
\end{tabular}

LSH $^{*}=$ Life Style healthful; NS** = Nutritional Supplements.

Table 2. Categorization of websites based on affiliation, specialisation and content type (Pearson's chi squared test).

\begin{tabular}{|c|c|c|c|c|}
\hline Categorisation & $\begin{array}{c}\text { Google }(n=21) \\
n(\%)\end{array}$ & $\begin{array}{c}\text { Yahoo }(\mathrm{n}=\mathbf{2 0}) \\
\mathrm{n}(\%)\end{array}$ & $\begin{array}{c}\text { MSN Search }(n=19) \\
n(\%)\end{array}$ & p-value \\
\hline \multicolumn{4}{|l|}{ Affiliation } & 0.647 \\
\hline Commercial & $9(42.9)$ & $12(60)$ & $11(57.9)$ & \\
\hline Non-profit organisation & $3(14.3)$ & $4(20)$ & $1(5.3)$ & \\
\hline University or medical centre & $7(33.3)$ & $3(15)$ & $6(31.6)$ & \\
\hline Government & $2(9.5)$ & $1(5)$ & $1(5.3)$ & \\
\hline \multicolumn{4}{|l|}{ Specialisation } & 0.857 \\
\hline Exclusively related to $\mathrm{OLT}^{*}$ & $3(14.3)$ & $2(10)$ & $3(15.8)$ & \\
\hline Part of the site dedicated OLT & $18(85.7)$ & $18(90)$ & $16(84.2)$ & \\
\hline \multicolumn{4}{|l|}{ Content type } & \\
\hline Medical facts & $13(61.9)$ & $14(70)$ & $12(63.2)$ & 0.845 \\
\hline Clinical trials & $20(95.2)$ & $19(95)$ & $18(94.7)$ & 0.997 \\
\hline Question and answer & $8(38.1)$ & $9(45)$ & $7(36.8)$ & 0.853 \\
\hline Human interest stories & $1(4.8)$ & $0(0)$ & $0(0)$ & 0.389 \\
\hline
\end{tabular}

OLT $^{*}=$ Oral leukoplakia treatment. 
Table 3. Website content based on JAMA benchmarks (Pearson's chi squared test).

\begin{tabular}{|l|c|c|c|c|}
\hline JAMA benchmarks & $\begin{array}{c}\text { Google (n=21) } \\
\mathbf{n}(\%)\end{array}$ & $\begin{array}{c}\text { Yahoo (n=20) } \\
\mathbf{n}(\%)\end{array}$ & $\begin{array}{c}\text { MSN Search (19) } \\
\mathbf{n}(\%)\end{array}$ & p-value \\
\hline Authorship & $9(42.8)$ & $9(45)$ & $14(73.7)$ & 0.098 \\
\hline Attribution & $7(33.3)$ & $5(25)$ & $5(26.3)$ & 0.816 \\
\hline Disclosure & $21(100)$ & $19(95)$ & $17(89.5)$ & 0.312 \\
\hline Currency & $17(80.9)$ & $13(65)$ & $11(57.9)$ & 0.272 \\
\hline
\end{tabular}

Table 4. Frequency distribution of overall scores achieved by websites using the DISCERN instrument (Kruskal-Wallis test).

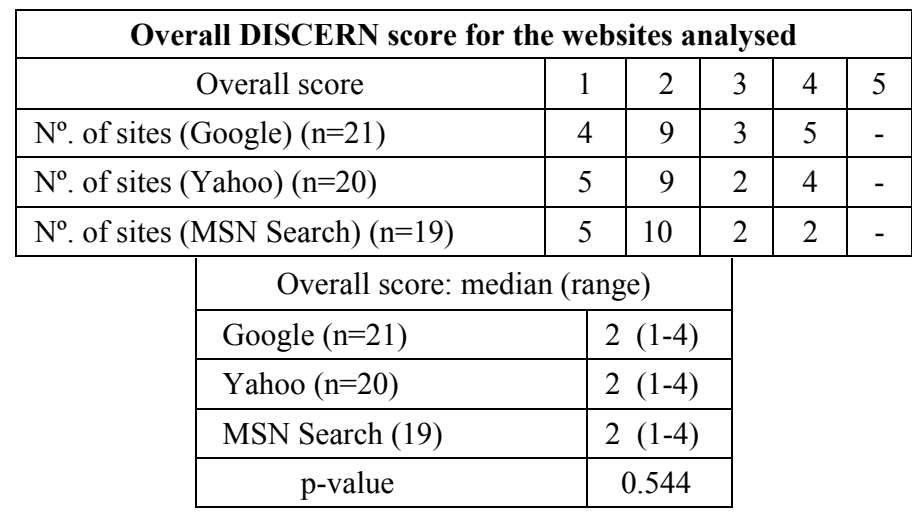

\section{Discusion}

The great amounts of information present in internet have implications for society on a global level.

In 2001, Risk et al. (17) retrieved over 60 million pages entering the word "health" in a generic search engine like Google. In a systematic review of 79 studies assessing the quality of health information for consumers on the Internet, $55(70 \%)$ were shown to have qualityrelated problems (14). Actually, there are no required standards for medical information on the Internet, in this sense some websites that appear to be educational are actually promotional in nature (18), while others may be inefficient, incomplete, out of date, difficult to understand, or contain conflicting information $(8,10)$. The results from this study demonstrate no difference between the three search engines (Google, Yahoo and MSN) for oral leukoplakia and the overall quality of websites was poor. Measuring quality is a complex problem and the same information may be appropriate or not depending on the personal needs of the individual searcher. Eysenbach et al. (14) indicated the need to apply standard evaluation tools to websites to assess their quality. The strategies proposed to evaluate the quality of medical information on the Internet can be grouped into different categories: codes of conduct, seals of guarnteed quality, user guides, government regulation, etc. However, actually there is no one tool (11).
In oral leukoplakia, the most common objective of care is to detect and to prevent malignant change. Several medial therapies have been suggested (anti-inflammatory agents, vitamins, cytotoxic agents,etc) and surgical treatments (scalpel, laser, cryoprobe, electrosurgery, photodynamic therapy, etc). Our results shows that the Internet information about oral leukoplakia for the patient, has low quality, little scientific and very heterogeneous. Most sites have a commercial content with very poor, useless or unrelated information, and are intended to promote sales.

In the present study, the sample of first 100 sites (top 100) may be considered as representative of the information that a regular user of Internet would visit; moreover, we used three different search engines and different quality instruments to evaluate the content of the web pages. In relation to the Health on the Net (HON) seal, only 4 Google sites (19.1\%), 4 Yahoo sites (20\%) and 2 MSN sites $(10.5 \%)$ presented this certification. Moreover, the Discern Instrument structure for assessing the sites was very helpful in orienting us to the criteria for assessing information. In our study, no page obtained maximum score (code 5) in any search engine.

The study carried out had certain limitations. For example, the search was carried out on one date ( 9 April, 2009) and may not be representative of matches form other search engines at other. The changes that the web- 
sites undergo, both as regards content, design and even services offered, are manifold; they may even change server or even country. This dynamic character may cause a website to change radically in very little time. Other limitations include the failure to identify other reputable websites, we study the first 100 sites (top 100), but only each search engine decides its first 100 sites.

The interplay of users to collaborate and deal with information overload has already proven successful in other areas outside the health space. For example, Wikipedia not only allows users to submit content on various topics, but also provides the capability for users to edit the content of others (19).

Therefore, Internet may rapidly disseminate false or biased medical information that is potentially dangerous to a large audience and expose the public to uncontrolled risks (10). There is no editorial control over medical information on the Internet because there is no control over the Internet in general. Nevertheless, in the scientific community, the function of editorial control is effected by a peer review process. The external accreditation of quality of health-related websites is not an easy task. It would be an enormous and costly task requiring a large staff with expertise in varying fields to monitor thousands of health-related sites; it is also vulnerable to fraudulent seals and claims, and may shut out small producers who are unable to afford accreditation fees. Finally, we must emphasize that more study is needed to work toward consensus in defining and measuring the quality of patient education on the Internet.

\section{References}

1. Warnakulasuriya S, Johnson NW, Van der Waal I. Nomenclature and classification of potentially malignant disorders of the oral mucosa. J Oral Pathol Med. 2007;36:575-80.

2. Fisher MA, Bouquot JE, Shelton BJ. Assessment of risk factors for oral leukoplakia in West Virginia. Community Dent Oral Epidemiol. 2005;33:45-52.

3. Kramer IR, Lucas RB, Pindborg JJ, Sobin LH. Definition of leukoplakia and related lesions: an aid to studies on oral precancer. Oral Surg Oral Med Oral Pathol. 1978;46:518-39.

4. Scheifele C, Reichart PA. Is there a natural limit of the transformation rate of oral leukoplakia? Oral Oncol. 2003;39:470-5.

5. Sciubba JJ. Oral leukoplakia. Crit Rev Oral Biol Med. 1995;6:14760.

6. Commission of the European Communities, Brussels. eEurope 2002: Quality Criteria for Health Related Websites. J Med Internet Res. 2002;4:E15.

7. Bernstam EV, Shelton DM, Walji M, Meric-Bernstam F. Instruments to assess the quality of health information on the World Wide Web: what can our patients actually use? Int J Med Inform. 2005;74:13-9.

8. Crocco AG, Villasis-Keever M, Jadad AR. Analysis of cases of harm associated with use of health information on the internet. JAMA. 2002;287:2869-71.

9. Kunst H, Groot D, Latthe PM, Latthe M, Khan KS. Accuracy of information on apparently credible websites: survey of five common health topics. BMJ. 2002;324:581-2.

10. Jadad AR, Gagliardi A. Rating health information on the Internet: navigating to knowledge or to Babel? JAMA. 1998;279:611-4.
11. Purcell GP, Wilson P, Delamothe T. The quality of health information on the internet. BMJ. 2002;324:557-8.

12. Boyer C, Gaudinat A, Baujard V, Geissbühler A. Health on the Net Foundation: assessing the quality of health web pages all over the world. Stud Health Technol Inform. 2007;129:1017-21.

13. Griffiths KM, Christensen H. Website quality indicators for consumers. J Med Internet Res. 2005;7:e55.

14. Eysenbach G, Powell J, Kuss O, Sa ER. Empirical studies assessing the quality of health information for consumers on the world wide web: a systematic review. JAMA. 2002;287:2691-700.

15. Bedell SE, Agrawal A, Petersen LE. A systematic critique of diabetes on the world wide web for patients and their physicians. Int $J$ Med Inform. 2004;73:687-94.

16. Charnock D, Shepperd S, Needham G, Gann R. DISCERN: an instrument for judging the quality of written consumer health information on treatment choices. J Epidemiol Community Health. 1999;53:105-11.

17. Risk A, Dzenowagis J. Review of internet health information quality initiatives. J Med Internet Res. 2001;3:E28.

18. Eysenbach G, Köhler C. How do consumers search for and appraise health information on the world wide web? Qualitative study using focus groups, usability tests, and in-depth interviews. BMJ. 2002;324:573-7.

19. Giles J. Wikipedia rival calls in the experts. Nature. 2006;443:493. 